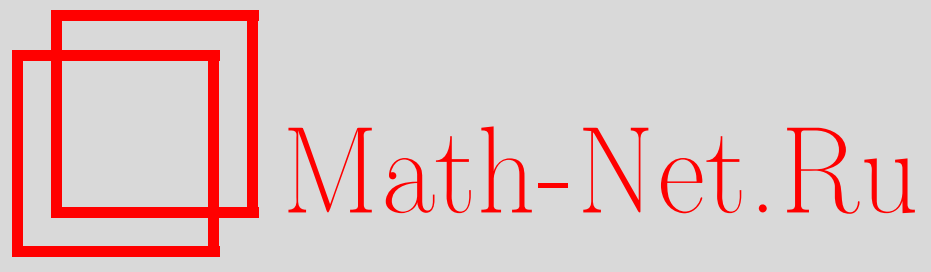

В. Г. Марихин, В. В. Соколов, Пары коммутирующих гамильтонианов, квадратичных по импульсам, ТМФ, 2006, том 149, номер 2, 147-160

DOI: https://doi.org/10.4213/tmf4224

Использование Общероссийского математического портала Math-Net.Ru подразумевает, что вы прочитали и согласны с пользовательским соглашением http://www . mathnet.ru/rus/agreement

Параметры загрузки:

IP : 52.6 .47 .48

26 апреля 2023 г., 07:50:20

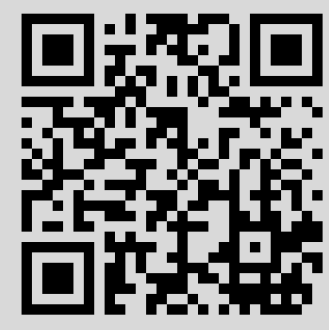




\section{ПАРЫ КОММУТИРУЮЩИХ ГАМИЛЬТОНИАНОВ, КВАДРАТИЧНЫХ ПО ИМПУЛЬСАМ}

В случае двух степеней свободы рассматриваются пары квадратичных по импульсам гамильтонианов, коммутирующих относительно стандартной скобки Пуассона. Найдены новые многопараметрические семейства таких пар. Приведена универсальная схема построения полного решения уравнения ГамильтонаЯкоби в терминах интегралов на некоторой алгебраической кривой. Для наиболее сложных примеров эта кривая является негиперэллиптическим накрытием над эллиптической кривой.

Ключевые слова: интегрируемые гамильтоновы системы, разделение переменных, алгебраические системы.

\section{1. ВВЕДЕНИЕ}

В работах [1]-[4] рассматривалась задача о парах квадратичных по импульсам гамильтонианов, коммутирующих относительно стандартной скобки Пуассона. Основной мотивацией данной работы является тот факт, что целый ряд моделей интегрируемых волчков, таких как волчок Шоттки-Манакова на $s o(4)$, волчок Клебша, гиростат Ковалевской и др., может быть сведен к задаче о паре коммутирующих гамильтонианов со стандартной скобкой Пуассона (см. [3] и приведенную там литературу). Более того, как показано в работе [3], задача о разделении переменных может быть эффективно решена в терминах канонического гамильтонова формализма, т.е. можно явно найти решение уравнения Гамильтона-Якоби соответствующей системы.

Целью данной работы является обобщение подхода, развитого в работе [3], на общий случай пар коммутирующих гамильтонианов с двумя степенями свободы, в том числе построение универсального решения уравнения Гамильтона-Якоби.

В разделе 2 сформулированы необходимые и достаточные условия существования указанных пар гамильтонианов, кратко рассмотрены свойства решений уравнения Эйлера-Дарбу, возникающего как одно из необходимых условий. В разделе 3

${ }^{*}$ Институт теоретический физики им. Л. Д. Ландау РАН, Москва, Россия. E-mail: mvg@itp.ac.ru, sokolov@itp.ac.ru 
построено универсальное решение уравнения Гамильтона-Якоби рассматриваемых систем в терминах интегралов на некоторой алгебраической кривой (в общем случае негиперэллиптической). В разделе 4 рассмотрены несколько классов решения уравнения Эйлера-Дарбу, которые приводят к построению коммутирующих пар гамильтонианов, и высказана гипотеза о том, что любая такая пара принадлежит к одному из этих классов. Найдены соответствующие алгебраические кривые для разделяющих переменных. Показано, что в случае моделей из классов 2 и 3 алгебраическая кривая является разветвленным негиперэллиптическим накрытием над эллиптической кривой. Пример применения общего подхода, развитого в данной работе, к физически интересному случаю волчка Стеклова содержится в приложении.

\section{2. ПАРЫ КВАДРАТИЧНЫХ ГАМИЛЬТОНИАНОВ}

Рассмотрим пару гамильтонианов вида

$$
\begin{aligned}
& H=a p_{1}^{2}+c p_{2}^{2}+d p_{1}+e p_{2}+f \\
& K=A p_{1}^{2}+C p_{2}^{2}+D p_{1}+E p_{2}+F
\end{aligned}
$$

коммутирующих относительно стандартной скобки Пуассона $\left\{p_{\alpha}, q_{\beta}\right\}=\delta_{\alpha \beta}$. Коэффициенты в формулах (1), (2) представляют собой некоторые (локально) аналитические функции переменных $q_{1}, q_{2}$.

Из условия $\{H, K\}=0$ следует, что

$$
a=\frac{S_{1}\left(q_{1}\right)}{\sigma_{1}\left(q_{1}\right)-\sigma_{2}\left(q_{2}\right)}, \quad c=\frac{S_{2}\left(q_{2}\right)}{\sigma_{1}\left(q_{1}\right)-\sigma_{2}\left(q_{2}\right)}
$$

для некоторых функций $S_{i}, \sigma_{i}$. Ясно, что в невырожденном случае $\sigma_{1}^{\prime} \neq 0, \sigma_{2}^{\prime} \neq 0$ функции $\sigma_{1}\left(q_{1}\right)$ и $\sigma_{2}\left(q_{2}\right)$ сводятся соответственно к $q_{1}$ и $q_{2}$ подходящим каноническим преобразованием вида

$$
\hat{q}_{1}=\phi\left(q_{1}\right), \quad \hat{q}_{2}=\phi\left(q_{2}\right), \quad \hat{p}_{1}=\frac{p_{1}}{\phi^{\prime}\left(q_{1}\right)}, \quad \hat{p}_{2}=\frac{p_{2}}{\phi^{\prime}\left(q_{2}\right)} .
$$

Одним из результатов работы [4] является

ТЕорема 1. Всякая пара коммутирующих гамильтонианов (1), (2) с $\sigma_{1}\left(q_{1}\right)=$ $q_{1}, \sigma_{2}\left(q_{2}\right)=q_{2}$ может быть сведена каноническим преобразованием

$$
\hat{p}_{1}=p_{1}+\frac{\partial F\left(q_{1}, q_{2}\right)}{\partial q_{1}}, \quad \hat{p}_{2}=p_{2}+\frac{\partial F\left(q_{1}, q_{2}\right)}{\partial q_{2}}
$$

$\kappa$ nаре вида

$$
H=\frac{U_{1}-U_{2}}{q_{1}-q_{2}}, \quad K=\frac{q_{2} U_{1}-q_{1} U_{2}}{q_{1}-q_{2}},
$$


где

$$
\begin{gathered}
U_{1}=S_{1}\left(q_{1}\right) p_{1}^{2}+\frac{\sqrt{S_{1}\left(q_{1}\right) S_{2}\left(q_{2}\right)} Z_{q_{1}}}{q_{1}-q_{2}} p_{2}-\frac{S_{1}\left(q_{1}\right) Z_{q_{1}}^{2}}{4\left(q_{1}-q_{2}\right)^{2}}+V_{1}\left(q_{1}, q_{2}\right), \\
U_{2}=S_{2}\left(q_{2}\right) p_{2}^{2}-\frac{\sqrt{S_{1}\left(q_{1}\right) S_{2}\left(q_{2}\right)} Z_{q_{2}}}{q_{1}-q_{2}} p_{1}-\frac{S_{2}\left(q_{2}\right) Z_{q_{2}}^{2}}{4\left(q_{2}-q_{1}\right)^{2}}+V_{2}\left(q_{1}, q_{2}\right), \\
V_{1}=\frac{1}{2} \sqrt{S_{1}\left(q_{1}\right)} \partial_{q_{1}}\left(\sqrt{S_{1}\left(q_{1}\right)} \frac{Z_{q_{1}}^{2}}{q_{1}-q_{2}}\right)+f_{1}\left(q_{1}\right), \\
V_{2}=\frac{1}{2} \sqrt{S_{2}\left(q_{2}\right)} \partial_{q_{2}}\left(\sqrt{S_{2}\left(q_{2}\right)} \frac{Z_{q_{2}}^{2}}{q_{2}-q_{1}}\right)+f_{2}\left(q_{2}\right)
\end{gathered}
$$

для некоторых функиий $Z\left(q_{1}, q_{2}\right), S_{i}\left(q_{i}\right)$ u $f_{i}\left(q_{i}\right)$. Скобка Пуассона $\{H, K\}$ равна нулю тогда и только тогда, когда

$$
Z_{q_{1}, q_{2}}=\frac{Z_{q_{1}}-Z_{q_{2}}}{2\left(q_{2}-q_{1}\right)}
$$

$u$

$$
\left(Z_{q_{1}} \frac{\partial}{\partial q_{2}}-Z_{q_{2}} \frac{\partial}{\partial q_{1}}\right)\left(\frac{V_{1}-V_{2}}{q_{1}-q_{2}}\right)=0
$$

ЗАмечание 1 . Функция $Z$ в формулах (4), (5) определена с точностью до сдвига $Z \rightarrow Z+k_{1}$. Функции $f_{i}$ в (5) определены с точностью до сдвига $f_{i}(x) \rightarrow f_{i}(x)+k_{2} x+$ $k_{3}$, где $k_{i}$ - произвольные постоянные. Этот сдвиг соответствует преобразованию $H \rightarrow H+k_{2}, K \rightarrow K+k_{3}$.

Отметим, что впервые уравнение Эйлера-Дарбу (6) в связи с задачей о паре коммутирующих квадратичных гамильтонианов возникло в работе [2].

Общее аналитическое решение уравнения Эйлера-Дарбу (6) имеет вблизи линии сингулярности $q_{1}=q_{2}$ следующее разложение:

$$
\begin{gathered}
Z\left(q_{1}, q_{2}\right)=A+\ln \left(q_{1}-q_{2}\right) B \\
A=\sum_{i=0}^{\infty} a_{i}\left(q_{1}+q_{2}\right)\left(q_{1}-q_{2}\right)^{2 i}, \quad B=\sum_{i=0}^{\infty} b_{i}\left(q_{1}+q_{2}\right)\left(q_{1}-q_{2}\right)^{2 i}
\end{gathered}
$$

где $a_{0}$ и $a_{1}$ - произвольные функции. Остальные коэффициенты могут быть легко выражены через эти две функции и их производные. Например, $b_{0}=a_{0}^{\prime \prime} / 2$.

Подставляя разложения функции $Z\left(q_{1}, q_{2}\right)$ в $(7)$, находим, что $B=0$. Нетрудно проверить, что всякое решение уравнения (6) с $B=0$ задается формулой

$$
Z\left(q_{1}, q_{2}\right)=z_{0}+\delta\left(q_{1}+q_{2}\right)+\left(q_{1}-q_{2}\right)^{2} \sum_{k=0}^{\infty} \frac{g^{(2 k)}\left(q_{1}+q_{2}\right)}{2^{2 k} k !(k+1) !}\left(q_{1}-q_{2}\right)^{2 k},
$$

где $g(x)$ - произвольная функция, а $z_{0}, \delta$ - произвольные постоянные. Будем называть функцию $g(x)$ производящей функиией для решения (8). Без потери общности положим $z_{0}=0$. Что касается $\delta$, то этот параметр играет важную роль при классификации гамильтонианов из теоремы 1. 
Опишем в замкнутом виде все функции $Z$, соответствующие рациональным производящим функциям $g$. Выбирая $g(x)=x^{n}$, получаем бесконечную последовательность полиномиальных решений $Z^{(n)}$ уравнения (6). В частности,

$$
\begin{gathered}
g(x)=1 \Longleftrightarrow Z^{(0)}(x, y)=(x-y)^{2}, \\
g(x)=x \Longleftrightarrow Z^{(1)}(x, y)=(x+y)(x-y)^{2}, \\
g(x)=x^{2} \Longleftrightarrow Z^{(2)}(x, y)=\frac{1}{4}\left((x-y)^{2}+4(x+y)^{2}\right)(x-y)^{2} .
\end{gathered}
$$

Вся последовательность может быть построена (с точностью до постоянных множителей) с помощью оператора

$$
x^{2} \frac{\partial}{\partial x}+y^{2} \frac{\partial}{\partial y}-\frac{1}{2}(x+y),
$$

действующего на $Z^{(0)}$. Рациональные функции $g(x)=(x-\mu)^{-n}$ порождают еще один класс точных решений уравнения (6). Например, имеем

$$
g_{\mu}(x)=\frac{1}{4} \frac{1}{x-2 \mu} \Longleftrightarrow Z_{\mu}(x, y)=\sqrt{(\mu-x)(\mu-y)}+\frac{1}{2}(x+y)-\mu .
$$

Решение, соответствующее полюсу порядка $n \geqslant 2$, может быть получено дифференцированием последней формулы по параметру $\mu$. Поскольку соответствие между функциями $Z$ и $g$ линейно, мы построили решение $Z$ с произвольной рациональной производящей функцией

$$
g(x)=\sum_{i} c_{i} x^{i}+\sum_{i, j} d_{i j}\left(x-\mu_{i}\right)^{-j} .
$$

ГиПотезА 1. Для любого гамильтониана (3)-(7) производящая функиия $g$ рациональна и имеет вид $g(x)=P(x) / S(x)$, где $P$ и $S$ - некоторые многочлены такие, чmo $\operatorname{deg} P<5, \operatorname{deg} S<6$.

В работах [2], [3] рассматривалось следующее решение системы (6), (7):

$$
\begin{gathered}
Z(x, y)=x+y, \quad S_{1}(x)=S_{2}(x)=\sum_{i=0}^{6} c_{i} x^{i}, \\
f_{1}(x)=f_{2}(x)=-\frac{3}{4} c_{6} x^{4}-\frac{1}{2} c_{5} x^{3}+\sum_{i=0}^{2} k_{i} x^{i},
\end{gathered}
$$

где $c_{i}, k_{i}$ - произвольные постоянные. В работе [3] показано, что волчок Клебша и $s o(4)$-волчок Шоттки-Манакова [5] являются частными случаями этой модели, и для этой модели найдено полное решение уравнения Гамильтона-Якоби. Полученные результаты могут рассматриваться как некоторое (обобщенное) разделение переменных на негиперэллиптической кривой рода 4.

Основная цель настоящей работы - построить полное решение уравнения Гамильтона-Якоби для произвольной пары гамильтонианов (3)-(7). 


\section{3. УНИВЕРСАЛЬНАЯ ФОРМУЛА ДЛЯ РЕШЕНИЯ УРАВНЕНИЯ ГАМИЛЬТОНА-ЯКОБИ}

Пусть гамильтонианы $H$ и $K$ имеют вид (3)-(5). Рассмотрим систему $H=e_{1}$, $K=e_{2}$, где $e_{i}$ - произвольные постоянные. Пусть $p_{1}=F_{1}(x, y), p_{2}=F_{2}(x, y)-$ ее решение. Здесь и далее мы используем обозначения $x$ и $y$ вместо $q_{1}$ и $q_{2}$. Из известной леммы Якоби следует, что если $\{H, K\}=0$, то $\partial F_{1} / \partial y=\partial F_{2} / \partial x$. Чтобы найти функцию действия $\mathrm{S}\left(x, y, e_{1}, e_{2}\right)$, достаточно в явном виде решить совместную систему

$$
\frac{\partial}{\partial x} \mathrm{~S}=F_{1}, \quad \frac{\partial}{\partial y} \mathrm{~S}=F_{2} .
$$

Перепишем формулы $H=e_{1}, K=e_{2}$ в виде

$$
p_{1}^{2}+a p_{2}+b=0, \quad p_{2}^{2}+A p_{1}+B=0,
$$

где

$$
\begin{aligned}
a=\frac{Z_{x}}{x-y} \sqrt{\frac{S_{2}(y)}{S_{1}(x)}}, \quad A=-\frac{Z_{y}}{x-y} \sqrt{\frac{S_{1}(x)}{S_{2}(y)}}, \\
b=-\frac{Z_{x}^{2}}{4(x-y)^{2}}+\frac{V_{1}-e_{1} x+e_{2}}{S_{1}(x)}, \quad B=-\frac{Z_{y}^{2}}{4(x-y)^{2}}+\frac{V_{2}-e_{1} y+e_{2}}{S_{2}(y)} .
\end{aligned}
$$

Легко проверить, что

$$
2 b_{y}+A a_{x}+2 a A_{x}=0, \quad 2 A a_{y}+a A_{y}+2 B_{x}=0 .
$$

С помощью уравнений (6) и (7) нетрудно получить также следующее тождество:

$$
A b_{x}-a B_{y}+2 A_{x} b-2 a_{y} B=0 .
$$

Используя стандартную технику резольвент Лагранжа (см., например, [6]), сведем систему (9), эквивалентную алгебраическому уравнению четвертой степени, к системе

$$
\begin{gathered}
u v=\frac{1}{4} a A, \\
A u^{3}+4 \frac{b}{a} u^{2} v-4 \frac{B}{A} u v^{2}-a v^{3}=0,
\end{gathered}
$$

которая эквивалентна кубическому уравнению относительно $u^{2}$. Пусть $\left(u_{k}, v_{k}\right), k=$ $1,2,3,-$ решения системы (12), (13) такие, что

$$
\begin{array}{cc}
u_{1}^{2}+u_{2}^{2}+u_{3}^{2}=-b, & v_{1}^{2}+v_{2}^{2}+v_{3}^{2}=-B, \\
u_{1} u_{2} u_{3}=-\frac{1}{8} a^{2} A, & v_{1} v_{2} v_{3}=-\frac{1}{8} A^{2} a .
\end{array}
$$


Тогда формулы

$$
\begin{array}{ll}
p_{1}=u_{1}+u_{2}+u_{3}, & p_{2}=v_{1}+v_{2}+v_{3} ; \\
p_{1}=u_{3}-u_{1}-u_{2}, & p_{2}=v_{3}-v_{1}-v_{2} ; \\
p_{1}=u_{2}-u_{1}-u_{3}, & p_{2}=v_{2}-v_{1}-v_{3} ; \\
p_{1}=u_{1}-u_{2}-u_{3}, & p_{2}=v_{1}-v_{2}-v_{3}
\end{array}
$$

определяют четыре решения системы (9). Для определенности будем рассматривать первое из них.

ЛЕмма 1. Для $i=1,2,3$ выполнены соотношения $\partial u_{i} / \partial y=\partial v_{i} / \partial x$.

ДокАзАтЕЛЬСтво. Дифференцируя уравнения (12) и (13) по $x$ и $y$, находим $u_{y}$ и $v_{x}$ как функции от $u$ и $v$. Тогда, выражая $v$ через $u$, получаем, что условие $u_{y}=v_{x}$ эквивалентно выполнению тождеств (10) и (11).

Лемма 1 означает, что в переменных $u_{1}, u_{2}, u_{3}$ происходит "частичное" разделение переменных. А именно, $\mathrm{S}=\mathrm{S}_{1}+\mathrm{S}_{2}+\mathrm{S}_{3}$, где $\mathrm{S}$ - искомая функция действия, а функции $\mathrm{S}_{i}$ определяются из системы

$$
\frac{\partial}{\partial x} S_{i}=u_{i}, \quad \frac{\partial}{\partial y} \mathbf{S}_{i}=v_{i}
$$

Положим

$$
u=\frac{1}{2} \frac{Z_{x}}{x-y} \sqrt{\frac{y-\xi}{x-\xi}}, \quad v=-\frac{1}{2} \frac{Z_{y}}{x-y} \sqrt{\frac{x-\xi}{y-\xi}} .
$$

Легко видеть, что пара $(u, v)$ для любого $\xi$ удовлетворяет уравнению (12). Кроме того, нетрудно проверить, что если $Z$ удовлетворяет (6), то $\partial u / \partial y=\partial v / \partial x$. Пользуясь этим фактом, введем функцию $\sigma(x, y, \xi)$ такую, что

$$
\frac{\partial \sigma}{\partial x}=u, \quad \frac{\partial \sigma}{\partial y}=v .
$$

Ясно, что функция $\sigma$ определена однозначно с точностью до прибавления произвольной функции от переменной $\xi$. В случае, когда производящая функция $g$ рациональна, соответствующая функция $Z$ выражается через квадратичные радикалы и $\sigma$ находится явно без каких-либо проблем. Положим $Y=\partial \sigma / \partial \xi$.

После умножения уравнения (13) на множитель

$$
-2 \frac{\sqrt{S_{1}(x)} \sqrt{S_{2}(y)} \sqrt{x-\xi} \sqrt{y-\xi}(x-y)}{Z_{x} Z_{y}}
$$

левая часть (13) может быть записана в виде

$$
-e_{2}+e_{1} \xi+\frac{y-\xi}{x-y}\left(V_{1}-\frac{S_{1}(x) Z_{x}^{2}}{4(x-\xi)(x-y)}\right)-\frac{x-\xi}{x-y}\left(V_{2}+\frac{S_{2}(y) Z_{y}^{2}}{4(y-\xi)(x-y)}\right) .
$$


ПРЕДЛОЖЕНИЕ. Пусть выполнены соотношения (6), (7). Тогда выражение (14) является функиией переменных $Y$ и

Доказательство. Обозначим функцию (14) через $\Psi(x, y, \xi)$. Рассмотрим якобиан

$$
J=\frac{\partial \Psi}{\partial x} \frac{\partial Y}{\partial y}-\frac{\partial \Psi}{\partial y} \frac{\partial Y}{\partial x}
$$

Заменяя $\partial Y / \partial y$ и $\partial Y / \partial x$ на $\partial v / \partial \xi$ и $\partial u / \partial \xi$, соответственно, нетрудно непосредственно проверить, что якобиан $J$ тождественно равен нулю в силу соотношений $(6),(7)$.

Согласно доказанному предложению соотношение $\Psi(x, y, \xi)=0$ может быть записано в виде $\phi(\xi, Y)=0$. Для практического нахождения функции $\phi$ полезно положить $y=x$. После этого выражение $Y(x, y, \xi)$ значительно упрощается и функция $\Psi(x, x, \xi)$ легко выражается через $\xi$ и $Y(x, x, \xi)$.

Уравнение $\phi(\xi, Y)=0$ задает кривую, на которой в терминах дифференциалов выражается функция действия $\mathrm{S}$.

Обозначим через $\xi_{k}(x, y), k=1,2,3$, корни кубического уравнения $\Psi(x, y, \xi)=0$.

Теорема 2. Функиия действия S имеет вид

$$
\mathrm{S}(x, y)=\sum_{k=1}^{3}\left(\sigma\left(x, y, \xi_{k}\right)-\int^{\xi_{k}} Y(\xi) d \xi\right)
$$

где $Y(\xi)$ - алгебраическая функция на кривой $\phi(\xi, Y)=0$.

ДокАЗАТЕЛЬСТво. Имеем

$$
\frac{\partial}{\partial x} \mathrm{~S}(x, y)=\sum_{k=1}^{3} \sigma_{x}\left(x, y, \xi_{k}\right)+\sum_{k=1}^{3}\left\{\sigma_{\xi}\left(x, y, \xi_{k}\right)-Y\left(\xi_{k}\right)\right\} \xi_{k, x}=\sum_{k=1}^{3} u_{k}=p_{1} .
$$

Аналогично

$$
\frac{\partial}{\partial y} \mathbf{S}(x, y)=p_{2}
$$

\section{4. ПРИМЕРЫ}

В этом разделе мы опишем все известные нам к настоящему моменту пары гамильтонианов (3)-(7). Для всех этих гамильтонианов в случае общего положения указывается явный вид функции $\sigma(x, y, \xi)$ и кривой $\phi(\xi, Y)=0$, задающих функцию действия (15). Вырождения нетрудно исследовать либо с помощью предельных переходов, либо применяя напрямую формулы (14), (15).

4.1. Класс 1. Для моделей из этого класса

$$
S_{1}=S_{2}=S, \quad f_{1}=f_{2}=f .
$$


TeOPEMA 3. Пусть

$$
g=\frac{\widetilde{G}}{S}, \quad \widetilde{G}=G-\frac{\delta}{10} S^{\prime}, \quad f=-\frac{4 \widetilde{G}^{2}}{S}-\frac{4 \delta}{3} \widetilde{G}^{\prime}-\frac{\delta^{2}}{12} S^{\prime \prime},
$$

¿əe

$$
S(x)=s_{5} x^{5}+s_{4} x^{4}+s_{3} x^{3}+s_{2} x^{2}+s_{1} x+s_{0}, \quad G(x)=g_{3} x^{3}+g_{2} x^{2}+g_{1} x+g_{0},
$$

$s_{i}, g_{i}, \delta$ - произвольные постоянные. Тогда функции $S, f$ и функция $Z$, соответствующая (см. раздел 2) производящей функиии g, удовлетворяют системе $(6),(7)$.

ЗамечаниЕ 2. Параметр $\delta$ из теоремы 3 совпадает с параметром $\delta$ из формулы (8). Предположим, что в формуле $(8) \delta=0$. Тогда можно проверить, что всякая пара гамильтонианов (3)-(7), (16), удовлетворяющая этому условию, описывается теоремой 3.

Рассмотрим случай общего положения

$$
S(x)=s_{5}\left(x-\mu_{1}\right)\left(x-\mu_{2}\right)\left(x-\mu_{3}\right)\left(x-\mu_{4}\right)\left(x-\mu_{5}\right),
$$

где $s_{5} \neq 0$ и все корни $\mu_{i}$ многочлена $S$ различны. Нетрудно проверить, что функция $Z$ для интегрируемой модели из теоремы 3 имеет вид

$$
Z(x, y)=\sum_{i=1}^{5} \nu_{i} \sqrt{\left(\mu_{i}-x\right)\left(\mu_{i}-y\right)}
$$

где $\nu_{i}$ - произвольные постоянные. Из формулы (8) коэффициенты $g_{i}$ и $\delta$ выражаются через постоянные $\nu_{i}$. Например, $2 \delta=-\sum \nu_{i}$. Функция $f$ задается формулой

$$
f(x)=-\frac{1}{16} \sum_{i=1}^{5} \nu_{i}^{2} \frac{S^{\prime}\left(\mu_{i}\right)}{x-\mu_{i}}+k_{1} x+k_{0},
$$

где $k_{1}, k_{0}$ - произвольные постоянные.

Вычисления показывают, что для функции (17)

$$
\begin{aligned}
\sigma(x, y, \xi) & =-\frac{1}{2} \sum_{i=1}^{5} \nu_{i} \ln \frac{\sqrt{x-\xi} \sqrt{y-\mu_{i}}+\sqrt{y-\xi} \sqrt{x-\mu_{i}}}{\sqrt{x-y} \sqrt{\mu_{i}-\xi}}, \\
Y & =\frac{1}{4} \sum_{i=1}^{N} \nu_{i} \frac{\sqrt{\left(x-\mu_{i}\right)\left(y-\mu_{i}\right)}}{\left(\xi-\mu_{i}\right) \sqrt{(x-\xi)(y-\xi)}} .
\end{aligned}
$$

Алгебраическая кривая имеет вид

$$
\phi(Y, \xi)=S(\xi) Y^{2}+f(\xi)-\xi e_{1}+e_{2}=0
$$

и является гиперэллиптической рода 2.

Оказывается (см. приложение), что волчок Стеклова на so(4) [7] является частным случаем модели из теоремы 3. 
4.2. Класс 2. Функции $Z$ для моделей этого класса являются специальными случаями функций $Z$ из класса 1 . Однако именно для этих специальных случаев функции $S$ и $f$ содержат гораздо больше произвольных параметров, чем обеспечивает общая формула из теоремы 3.

Такие функции $Z$ могут быть определены как решения системы

$$
Z_{x y}=\frac{Z_{x}-Z_{y}}{2(y-x)}=\frac{1}{3} U(Z) Z_{x} Z_{y},
$$

где $U$ - некоторая функция одной переменной.

ЗАмЕчаниЕ 3. Можно проверить, что этот класс решений уравнения ЭйлераДарбу $Z_{x y}=\left(Z_{x}-Z_{y}\right) /(2(y-x))$ совпадает с классом решений вида

$$
Z=F\left(\frac{h(x)-h(y)}{x-y}\right),
$$

где $F$ и $h$ - некоторые функции одной переменной. При этом оказывается, что $U=F^{\prime \prime} / F^{\prime 2}$.

Лемма 2. Система (19) совместна тогда и толъко тогда, когда

$$
U=\frac{3}{2} \frac{B^{\prime}}{B}, \quad B(Z)=b_{2} Z^{2}+b_{1} Z+b_{0},
$$

где $b_{i}-$ произвольные постоянные.

В случае $\operatorname{deg} B=2$ с точностью до линейных замен $Z$ можно считать, что

$$
Z(x, y)=\sqrt{\left(x-\mu_{1}\right)\left(y-\mu_{1}\right)}+\sqrt{\left(x-\mu_{2}\right)\left(y-\mu_{2}\right)} .
$$

При этом $b_{2}=1, b_{1}=0, b_{0}=-\left(\mu_{1}-\mu_{2}\right)^{2}$.

Если $\operatorname{deg} B=1$, то

$$
Z(x, y)=\sqrt{x y}+\frac{1}{2}(x+y)
$$

$b_{1}=1, b_{2}=b_{0}=0$.

Наконец, если $\operatorname{deg} B=0$, то

$$
Z(x, y)=x+y \text {. }
$$

Этот случай был рассмотрен в работе [3].

Исследуем теперь эти три случая подробно.

1. Пусть функция $Z$ задается формулой (20). Тогда

$S(x)=\left(x-\mu_{1}\right)\left(x-\mu_{2}\right) P(x)+\left(x-\mu_{1}\right)^{3 / 2}\left(x-\mu_{2}\right)^{3 / 2} Q(x), \quad \operatorname{deg} P \leqslant 3, \quad \operatorname{deg} Q \leqslant 2$,

И

$$
\begin{aligned}
f(x)=f_{0} & +f_{1} x+k_{2}\left(x-\mu_{1}\right)^{1 / 2}\left(x-\mu_{2}\right)^{1 / 2}+\frac{\mu_{2}-\mu_{1}}{16}\left(\frac{P\left(\mu_{1}\right)}{x-\mu_{1}}-\frac{P\left(\mu_{2}\right)}{x-\mu_{2}}\right)+ \\
+ & \frac{\mu_{2}-\mu_{1}}{32}\left(x-\mu_{1}\right)^{1 / 2}\left(x-\mu_{2}\right)^{1 / 2}\left(\frac{Q\left(\mu_{1}\right)}{x-\mu_{1}}-\frac{Q\left(\mu_{2}\right)}{x-\mu_{2}}\right) .
\end{aligned}
$$


В случае, когда $Q=0, k_{2}=0$, эти формулы совпадают с соответствующими формулами для класса 1 . Функции $\sigma, Y$ задаются формулами, аналогичными формулам (18) для класса 1:

$$
\begin{aligned}
\sigma(x, y, \xi) & =-\frac{1}{2} \sum_{i=1}^{2} \ln \frac{\sqrt{x-\xi} \sqrt{y-\mu_{i}}+\sqrt{y-\xi} \sqrt{x-\mu_{i}}}{\sqrt{x-y} \sqrt{\mu_{i}-\xi}}, \\
Y & =\frac{1}{4} \sum_{i=1}^{2} \frac{\sqrt{\left(x-\mu_{i}\right)\left(y-\mu_{i}\right)}}{\left(\xi-\mu_{i}\right) \sqrt{(x-\xi)(y-\xi)}} .
\end{aligned}
$$

Алгебраическую кривую в этом случае можно записать в виде

$$
\left[S_{R}(\xi)+\eta S_{I}(\xi)\right] Y^{2}-\left[k_{R}(\xi)+\eta k_{I}(\xi)\right]=0,
$$

где

$$
\begin{aligned}
S_{R}(x) & =\left(x-\mu_{1}\right)\left(x-\mu_{2}\right) P(x), \quad S_{I}(x)=\left(x-\mu_{1}\right)\left(x-\mu_{2}\right) Q(x), \\
k_{R}(x) & =-e_{2}+e_{1} x-f_{0}-f_{1} x-\frac{\mu_{2}-\mu_{1}}{16}\left(\frac{P\left(\mu_{1}\right)}{x-\mu_{1}}-\frac{P\left(\mu_{2}\right)}{x-\mu_{2}}\right), \\
k_{I}(x) & =k_{2}-\frac{1}{32}\left(\mu_{1}-\mu_{2}\right)^{2}-\frac{\mu_{1}-\mu_{2}}{16}\left(\frac{Q\left(\mu_{1}\right)}{x-\mu_{1}}-\frac{Q\left(\mu_{2}\right)}{x-\mu_{2}}\right) \\
\frac{1}{\eta} & =\frac{1}{\sqrt{\xi-\mu_{1}} \sqrt{\xi-\mu_{2}}} \sqrt{1-\frac{\left(\mu_{1}-\mu_{2}\right)^{2}}{16\left(\xi-\mu_{1}\right)^{2}\left(\xi-\mu_{2}\right)^{2} Y^{2}}} .
\end{aligned}
$$

Отметим, что из последней формулы можно выразить $\eta^{2}$ как функцию $\xi, Y$ и после простых преобразований привести уравнение для кривой к стандартной алгебраической форме

$$
\Phi(\xi, Y)=\sum_{i, j} c_{i j} Y^{i} \xi^{j}=0 .
$$

Однако ответ получается довольно громоздким.

Выражая $Y$ как функцию от $(\xi, \eta)$ и подставляя в $(23)$, можно проверить, что в переменных $(\xi, \eta)$ эта кривая приобретает вид произвольной 10-параметрической кубики. Таким образом, в случае общего положения кривая $\phi(Y, \xi)=0$, в терминах которой записывается функция действия (см. теорему 2), является накрытием над эллиптической кривой. Поскольку

$$
\eta=\left(\xi-\mu_{1}\right)\left(\frac{\sqrt{x-\mu_{1}}}{\sqrt{x-\mu_{2}}}+\frac{\sqrt{y-\mu_{1}}}{\sqrt{y-\mu_{2}}}\right)^{-1}+\left(\xi-\mu_{2}\right)\left(\frac{\sqrt{x-\mu_{2}}}{\sqrt{x-\mu_{1}}}+\frac{\sqrt{y-\mu_{2}}}{\sqrt{y-\mu_{1}}}\right)^{-1},
$$

то точки $\left(\xi_{1}, \eta_{1}\right),\left(\xi_{2}, \eta_{2}\right),\left(\xi_{3}, \eta_{3}\right)$ лежат на одной прямой.

2. Для функции $Z$ вида (21) имеем

$$
\begin{aligned}
& S(x)=x P(x)+x^{3 / 2} Q(x), \quad \operatorname{deg} P \leqslant 3, \quad \operatorname{deg} Q \leqslant 2, \\
& f(x)=-\frac{1}{16 x} P(x)-\frac{1}{32 \sqrt{x}} Q(x)+f_{1} x+f_{q} \sqrt{x}+f_{0} .
\end{aligned}
$$


Функция $Y$ задается формулой

$$
Y=\frac{\xi+\sqrt{x} \sqrt{y}}{4 \xi \sqrt{x-\xi} \sqrt{y-\xi}}
$$

Кривая и в этом случае может быть записана в виде (23), где

$$
\begin{gathered}
S_{R}(x)=x P(x), \quad S_{I}(x)=x Q(x), \\
k_{R}(x)=-e_{2}+e_{1} x-f_{0}-f_{1} x+\frac{1}{16 x} P(x), \quad k_{I}(x)=\frac{1}{16 x} Q(x)-f_{q}, \\
\eta=\frac{4 Y \xi^{3 / 2}}{\sqrt{16 Y^{2} \xi^{2}-1}} .
\end{gathered}
$$

В переменных $(\xi, \eta)$ она также имеет вид произвольной кубики. Из формулы $\eta=$ $(\xi+\sqrt{x y}) /(\sqrt{x}+\sqrt{y})$ следует, что точки $\left(\xi_{1}, \eta_{1}\right),\left(\xi_{2}, \eta_{2}\right),\left(\xi_{3}, \eta_{3}\right)$ лежат на одной прямой.

3. Для функции $Z$, заданной формулой (22), имеем

$$
\begin{aligned}
& S(x)=s_{6} x^{6}+s_{5} x^{5}+s_{4} x^{4}+s_{3} x^{3}+s_{2} x^{2}+s_{1} x+s_{0}, \\
& f(x)=-\frac{1}{40} S^{\prime \prime}(x)-\frac{1}{32 \sqrt{x}} Q(x)+f_{2} x^{2}+f_{1} x+f_{0} .
\end{aligned}
$$

В этом случае

$$
Y=\frac{1}{2 \sqrt{x-\xi} \sqrt{y-\xi}} .
$$

Кривая имеет относительно простой алгебраический вид:

$S(\xi) Y^{6}-F(\xi) Y^{4}-\left(\frac{1}{8} F^{\prime \prime}(\xi)+\frac{7}{1920} S^{I V}(\xi)-\frac{k_{2}}{2}\right) Y^{2}-\frac{s_{6}}{64}=0, \quad F(\xi)=-e_{2}+e_{1} \xi-f(\xi)$,

и в переменных $(\xi, \eta)$, где $\eta=\xi^{2}-1 /\left(4 Y^{2}\right)$, имеет вид произвольной кубики. Поскольку $\eta=\xi(x+y)-x y$, то точки $\left(\xi_{1}, \eta_{1}\right),\left(\xi_{2}, \eta_{2}\right),\left(\xi_{3}, \eta_{3}\right)$ лежат на одной прямой.

4.3. Класс 3. Гамильтониан (3)-(7) назовем несимметрическим, если $S_{1}(x) \neq$ $S_{2}(x)$, или $f_{1}(x) \neq f_{2}(x)$.

Теорема 4 [4]. В несимметрическом случае функиии $Z, S_{i}, f_{i}$ удовлетворяют (6), (7) тогда и только тогда, когда

$$
\delta=0, \quad g=\frac{1}{H}, \quad S_{1,2}=W H \pm M H^{3 / 2}, \quad f_{1,2}=-\frac{4 W}{H} \mp 2 M H^{-1 / 2} \pm a H^{1 / 2},
$$

где $g$ - порождающая функиия для $Z$,

$$
\begin{gathered}
W(x)=w_{3} x^{3}+w_{2} x^{2}+w_{1} x+w_{0}, \quad H(x)=h_{2} x^{2}+h_{1} x+h_{0}, \\
M(x)=m_{2} x^{2}+m_{1} x+m_{0},
\end{gathered}
$$

$w_{i}, h_{i}, m_{i}, a$ - произвольные постоянные. 
Рассмотрим случай общего положения $H(x)=\left(x-\mu_{1}\right)\left(x-\mu_{2}\right)$. Алгебраическая кривая в этом случае задается формулой

$$
\begin{aligned}
\Psi(\xi, Y) & =-e_{2}+e_{1} \xi-\frac{R W(\xi)}{2\left(\xi-\mu_{1}\right)\left(\xi-\mu_{2}\right)\left(\mu_{2}-\mu_{1}\right)}+ \\
& +4 M(\xi) \sqrt{2} Y \frac{\sqrt{\xi-\mu_{1}} \sqrt{\xi-\mu_{2}}}{\left(\mu_{2}-\mu_{1}\right)^{3 / 2}} \sqrt{R}+8 b \sqrt{2} Y \frac{\left(\xi-\mu_{1}\right)^{3 / 2}\left(\xi-\mu_{2}\right)^{3 / 2}}{\sqrt{R} \sqrt{\mu_{2}-\mu_{1}}}=0
\end{aligned}
$$

где

$$
\begin{aligned}
& Y=\frac{\sqrt{\left(x-\mu_{1}\right)\left(y-\mu_{1}\right)}}{\left(\xi-\mu_{1}\right) \sqrt{(x-\xi)(y-\xi)}}-\frac{\sqrt{\left(x-\mu_{2}\right)\left(y-\mu_{2}\right)}}{\left(\xi-\mu_{2}\right) \sqrt{(x-\xi)(y-\xi)}}, \\
& R=16\left(\xi-\mu_{1}\right)^{2}\left(\xi-\mu_{2}\right)^{2} Y^{2}-\left(\mu_{1}-\mu_{2}\right)^{2} .
\end{aligned}
$$

Подставляя

$$
Y=\frac{1}{4} \frac{\left(\mu_{1}-\mu_{2}\right)^{3 \eta / 2}}{\left(\xi-\mu_{2}\right)\left(\xi-\mu_{1}\right) \sqrt{\eta^{2}\left(\mu_{2}-\mu_{1}\right)-8\left(\xi-\mu_{1}\right)\left(\xi-\mu_{2}\right)}}
$$

в (24), получаем кубику в переменных $(\xi, \eta)$ с полным набором из десяти независимых параметров. Нетрудно проверить, что $\eta=a(x, y) \xi+b(x, y)$, где $a, b$ - некоторые функции.

Таким образом, в случае моделей из классов 2 и 3 алгебраическая кривая является разветвленным негиперэллиптическим накрытием над эллиптической кривой. Динамика трех точек $\left(\xi_{1}, Y_{1}\right),\left(\xi_{2}, Y_{2}\right),\left(\xi_{3}, Y_{3}\right)$ на этой кривой (см. теорему 2) подчиняется следующему условию: их проекции на эллиптическую базу $\left(\xi_{1}, \eta_{1}\right),\left(\xi_{2}, \eta_{2}\right)$, $\left(\xi_{3}, \eta_{3}\right)$ лежат на одной прямой.

ГиПотезА 2. Всякая пара гамильтонианов (3)-(7) принадлежит $к$ одному из трех классов, описанных выше.

\section{Волчок Стеклова}

Покажем, что случай волчка Стеклова на so(4) после ограничения на симплектические листы является частным случаем модели из класса 1. Гамильтониан и дополнительный интеграл в случае волчка Стеклова имеют вид

$$
H=\left(\mathbf{S}_{1}, A \mathbf{S}_{1}\right)+\left(\mathbf{S}_{1}, B \mathbf{S}_{2}\right), \quad K=\left(\mathbf{S}_{1}, \bar{A} \mathbf{S}_{1}\right)+\left(\mathbf{S}_{1}, \bar{B} \mathbf{S}_{2}\right),
$$

где

$$
\begin{array}{ll}
A=-\alpha^{2} \operatorname{diag}\left(\frac{1}{\alpha_{1}^{2}}, \frac{1}{\alpha_{2}^{2}}, \frac{1}{\alpha_{3}^{2}}\right), & B=\alpha \operatorname{diag}\left(\alpha_{1}, \alpha_{2}, \alpha_{3}\right), \\
\bar{A}=-\operatorname{diag}\left(\alpha_{1}^{2}, \alpha_{2}^{2}, \alpha_{3}^{2}\right), & \bar{B}=\alpha \operatorname{diag}\left(\frac{1}{\alpha_{1}}, \frac{1}{\alpha_{2}}, \frac{1}{\alpha_{3}}\right),
\end{array}
$$


$\alpha=\alpha_{1} \alpha_{2} \alpha_{3}$. Здесь $\mathbf{S}_{i}$ - трехмерные векторы с компонентами $S_{i}^{\alpha}$. Легко проверить, что гамильтонианы $H$ и $K$ коммутируют относительно спиновой скобки Пуассона

$$
\left\{S_{i}^{\alpha}, S_{j}^{\beta}\right\}=\kappa \varepsilon_{\alpha \beta \gamma} \delta_{i j} S_{i}^{\gamma}
$$

Нам удобно положить постоянную $\kappa$ равной $-2 i$.

Фиксируем значения функций Казимира для спиновой скобки: $\left(\mathbf{S}_{k}, \mathbf{S}_{k}\right)=j_{k}^{2}$, $k=1,2$. Тогда формулы

$$
\mathbf{S}_{k}=\pi_{k} \mathbf{K}\left(Q_{k}\right)+\frac{j_{k}}{2} \mathbf{K}^{\prime}\left(Q_{k}\right), \quad \mathbf{K}(Q)=\left(\left(Q^{2}-1\right), i\left(Q^{2}+1\right), 2 Q\right),
$$

определяют координаты Дарбу $\pi_{1}, \pi_{2}, Q_{1}, Q_{2}$ для симплектического листа пуассонова многообразия с координатами $\mathbf{S}_{k}, k=1,2$. Поскольку это преобразование линейно по импульсам $\pi_{k}$, в результате получается пара коммутирующих относительно скобки $\left\{\pi_{\alpha}, Q_{\beta}\right\}=\delta_{\alpha \beta}$ гамильтонианов, квадратичных по импульсам. Укажем канонические преобразования, приводящие эту пару к виду (3)-(5), (16).

В результате канонического преобразования

$$
P_{1}=\pi_{1} \sqrt{r\left(Q_{1}\right)}, \quad P_{2}=\pi_{2} \sqrt{R\left(Q_{2}\right)}, \quad d X=\frac{d Q_{1}}{\sqrt{r\left(Q_{1}\right)}}, \quad d Y=\frac{d Q_{2}}{\sqrt{R\left(Q_{2}\right)}},
$$

где

$$
r\left(Q_{1}\right)=\left(\mathbf{K}\left(Q_{1}\right), A \mathbf{K}\left(Q_{1}\right)\right), \quad R\left(Q_{2}\right)=\left(\mathbf{K}\left(Q_{2}\right), \bar{A} \mathbf{K}\left(Q_{2}\right)\right)
$$

получаем

$$
\begin{aligned}
& H=P_{1}^{2}+2 P_{1} P_{2} V+j_{2} P_{1} V_{Y}+j_{1} P_{2} V_{X}+\frac{1}{2} j_{1} j_{2} V_{X, Y}+\frac{j_{1}^{2}}{6}\left(\frac{g_{1}^{\prime \prime}(X)}{g_{1}(X)}-\frac{3}{2}\left(\frac{g_{1}^{\prime}(X)}{g_{1}(X)}\right)^{2}\right), \\
& K=P_{2}^{2}+2 P_{1} P_{2} W+j_{2} P_{1} W_{Y}+j_{1} P_{2} W_{X}+\frac{1}{2} j_{1} j_{2} W_{X, Y}+\frac{j_{2}^{2}}{6}\left(\frac{g_{2}^{\prime \prime}(Y)}{g_{2}(Y)}-\frac{3}{2}\left(\frac{g_{2}^{\prime}(Y)}{g_{2}(Y)}\right)^{2}\right) .
\end{aligned}
$$

Здесь

$$
V(X, Y)=\frac{\left(\mathbf{K}\left(Q_{1}\right), B \mathbf{K}\left(Q_{2}\right)\right)}{\sqrt{r\left(Q_{1}\right)} \sqrt{R\left(Q_{2}\right)}}, \quad W(X, Y)=\frac{\left(\mathbf{K}\left(Q_{1}\right), \bar{B} \mathbf{K}\left(Q_{2}\right)\right)}{\sqrt{r\left(Q_{1}\right)} \sqrt{R\left(Q_{2}\right)}},
$$

$g_{1}(X)=\sqrt{r\left(Q_{1}\right)}, g_{2}(Y)=\sqrt{R\left(Q_{2}\right)}$. Применяя теперь каноническое преобразование $\left(P_{1}, P_{2}, X, Y\right) \rightarrow\left(p_{1}, p_{2}, x, y\right)$ вида

$$
\begin{aligned}
d X & =\frac{1}{2}\left(\frac{d x}{\sqrt{S(x)}}+\frac{d y}{\sqrt{y S(y)}}\right), \quad d Y=-\frac{1}{2}\left(\frac{d x}{\sqrt{x S(x)}}+\frac{d y}{\sqrt{S(y)}}\right) \\
P_{1} & =\frac{2}{\sqrt{x}-\sqrt{y}}\left[\left(p_{1}-\frac{j_{1}+j_{2}}{4(x-y)} \sqrt{\frac{y}{x}}\right) \sqrt{S(x)}-\left(p_{2}+\frac{j_{1}+j_{2}}{4(x-y)} \sqrt{\frac{x}{y}}\right) \sqrt{S(y)}\right] \\
P_{2} & =\frac{2}{\sqrt{x}-\sqrt{y}}\left[\left(p_{1}-\frac{j_{1}+j_{2}}{4(x-y)} \sqrt{\frac{y}{x}}\right) \sqrt{y S(x)}-\left(p_{2}+\frac{j_{1}+j_{2}}{4(x-y)} \sqrt{\frac{x}{y}}\right) \sqrt{x S(y)}\right],
\end{aligned}
$$


получим (3)-(5), (16), где

$$
\begin{gathered}
S(x)=-4 x\left(1+\alpha_{1}^{2} x\right)\left(1+\alpha_{2}^{2} x\right)\left(1+\alpha_{3}^{2} x\right), \quad Z(x, y)=-\frac{1}{2} j_{1}(x+y)-j_{2} \sqrt{x y} \\
f(x)=\frac{1}{4}\left(j_{1}^{2} \alpha^{2} x^{2}+\frac{j_{2}^{2}}{x}\right)-j_{2}^{2} \frac{1}{4} \alpha^{2}\left(\frac{1}{\alpha_{1}^{2}}+\frac{1}{\alpha_{2}^{2}}+\frac{1}{\alpha_{3}^{2}}\right) x .
\end{gathered}
$$

Благодарности. Авторы благодарны Е. В. Ферапонтову за внимание к работе. Исследования были частично поддержаны РФФИ (гранты № 05-01-00189 и № 04-0100403), а также Программой поддержки ведущих научных школ (НШ-1716.2003.1, НШ-6358.2006.2).

\section{Список литературы}

[1] B. Dorizzi, B. Grammaticos, A. Ramani, P. Winternitz, J. Math. Phys., 26 (1985), 30703079; E. V. Ferapontov, A. P. Fordy, Physica D, 108 (1997), 350-364; Rep. Math. Phys., 44:1-2 (1999), 71-80; E. McSween, P. Winternitz, J. Math. Phys., 41 (2000), 2957-2967.

[2] H. M. Yehia, J. Phys. A, 25 (1992), 197-221.

[3] V. G. Marikhin, V. V. Sokolov, Regul. Chaotic Dyn., 10:1 (2005), 59-70.

[4] В. Г. Марихин, В. В. Соколов, УМН, 60:5 (2005), 175-176.

[5] F. Schottky, Sitzungsber. König. Preuss. Acad. Wiss. zu Berlin, 13 (1891), 227-232; С. В. Манаков, Функи. анализ и его прилож., 10:4 (1976), 93-94; A. Clebsch, Math. Ann., 3 (1870), 238-262.

[6] В. В. Прасолов, Ю. П. Соловьев, Эллиптические функиии и алгебраические уравнения, Факториал, М., 1997.

[7] V.A. Stekloff, Ann. Fac. Sci. Toulouse Sci. Math. Sci. Phys., Ser. 3, 1 (1909), 145-226.

Поступила в редакцию 24.05.2006 